\title{
A Range-free Localization of Passive RFID Tags Using Mobile Readers
}

\author{
Jiaqing Luo, Shijie Zhou, Hongrong Cheng, Yongjian Liao \\ School of Computer Science and Engineering \\ University of Electronic Science and Technology of China \\ Chengdu, Sichuan, China \\ Email: csjluo@hotmail.com, \{sjzhou, hrcheng, liaoyj\}@uestc.edu.cn
}

\author{
Kai Bu \\ College of Computer Science and Technology \\ Zhejiang University \\ Hangzhou, Zhejiang, China \\ Email: kaibu@zju.edu.cn
}

\begin{abstract}
Recently, there has been growing interest in indoor localization, because numerous applications depend on the rapid and accurate position estimation of tagged objects. While RFIDbased indoor localization is attractive, the need for a large-scale and high-density deployment of readers and reference tags is costly. Being the range-free localization, our schemes depend solely on mobile readers without reference tags or other devices, and it avoids the need of distance estimation according to RSSI or phase difference. We propose two novel algorithms, continuous scanning and category-based scheduling, for locating single and multiple tagged objects, respectively. Our primary experimental results show that the system can achieve high time efficiency and localization accuracy.
\end{abstract}

\section{INTRODUCTION}

Most existing RFID-based localization systems rely on Received Signal Strength Indicator (RSSI) measurements that may face some practical issues: 1) RSSI functions are not available on most RFID devices. 2) The deployment of highdensity reference tags and multiple readers is costly. 3) The interference among reference tags may change signal strength, and affect localization accuracy [1], [2]. 4) RSSI measurements are commonly used for locating active tags [1], [3].

In this paper, we design and implement a cost-effective RFID indoor localization system for locating objects attached with passive tags. In particular, we deploy two guide rails, $x$-axis and $y$-axis guide rails, on the ceiling of a warehouse. These guide rails can move a reader along either the $x$-axis or $y$-axis. We then propose a continuous scanning algorithm to improve the accuracy for locating a single tagged object. Such an algorithm records the locations of the reader when the first and the last times it successfully detects the tagged object. The location of the tagged object is at one of the intersection points of two circles that centered at the recorded locations with a radius equal to the read range. We also propose a categorybased scheduling algorithm to shorten the time for locating multiple tagged objects placed in different storage areas. When localization requests arrive, the number of storage areas to be scanned may change with time. Thus, the problem of finding an optimal scan path for the reader is a time-dependent optimization problem, which can be modeled by the dynamic traveling salesman problem (DTSP). The proposed algorithm decomposes DTSP into a series of static TSPs, and runs the 2-approximation algorithm to solve the static TSP at regular time intervals. Our preliminary experimental results show that, under the given condition, the continuous scanning can reduce $30 \%$ localization error and save $10 \%$ localization time. The category-based scheduling can shorten $42 \%$ movement time of the reader.

\section{SYSTEM DESIGN AND IMPLEMENTATION}

We take the following four steps to build the architecture of the system. 1) We firstly customize two kinds of guide rails, $x$-axis and $y$-axis guide rails, according to the size of the warehouse ceiling. 2) We then install two $y$-axis guide rails on both vertical edges of the warehouse ceiling. 3) After that, we install the $x$-axis guide rail between those two $y$-axis guide rails. 4) Finally, we attach a reader on the $x$-axis guide rail. Two electric motors installed on guide rails provide power to move the reader from one location to another. Figures 1 shows our prototype system.

\section{LOCALIZATION ALGORITHM}

The continuous scanning algorithm is range-free. We set the lengths of $x$-axis and $y$-axis guide rails to $2 r+W$ and $\left\lceil\frac{L-r}{2 r}\right\rceil 2 r$, respectively. As shown in Figure 2 , the reader firstly moves from $(-r, r)$ to $(r+W, r)$ along the $x$-axis. When it arrives at $(r+W, r)$, it moves $2 r$ along the $y$-axis and then goes to the negative $x$-axis. It continually scans the tagged object while moving along guide rails, and records its location when it successfully detects the tagged object. Suppose that the reader locates at $(a, b)$ and $\left(a^{\prime}, b\right)$ when the first and last times it successfully detects the tagged tag. Here, $-2 r \leq a^{\prime}-$ $a \leq 2 r$ and $b=(2 n+1) r$ for $n=0,1,2, \ldots,\left\lfloor\frac{L}{2 r}\right\rfloor$. The tagged object must locate at one point of intersection of two circles with same radius $r$, centered at $(a, b)$ and at $\left(a^{\prime}, b\right)$, respectively. The tagged object may locate at either $\left(x_{1}, y_{1}\right)$ or $\left(x_{2}, y_{2}\right)$. To eliminate the localization ambiguity, the reader moves $\frac{y_{1}-y_{2}}{2}$ along the $y$-axis. If it can still detect the tagged object, the tagged object is located at $\left(x_{1}, y_{1}\right)$. Otherwise, the tagged object is located at $\left(x_{2}, y_{2}\right)$.

\section{SCHEDULING ALGORITHM}

Products or goods with the same category are usually placed in the same storage area. To handle multiple requests, the reader may scan different storage areas. The problem of finding the optimal scan path for the reader can be modeled 


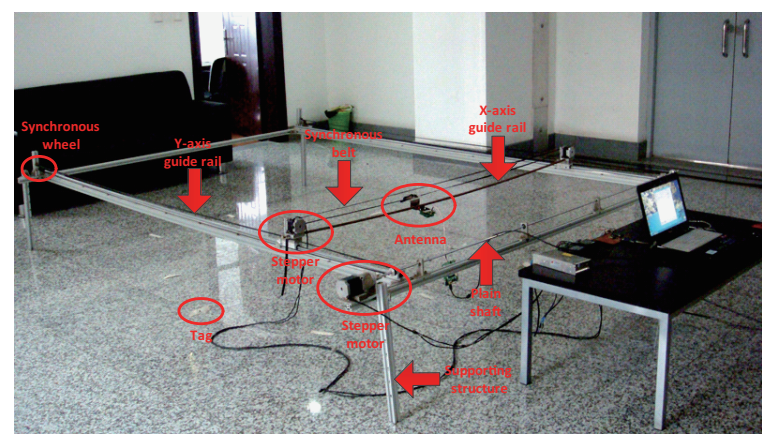

Fig. 1. The prototype system.

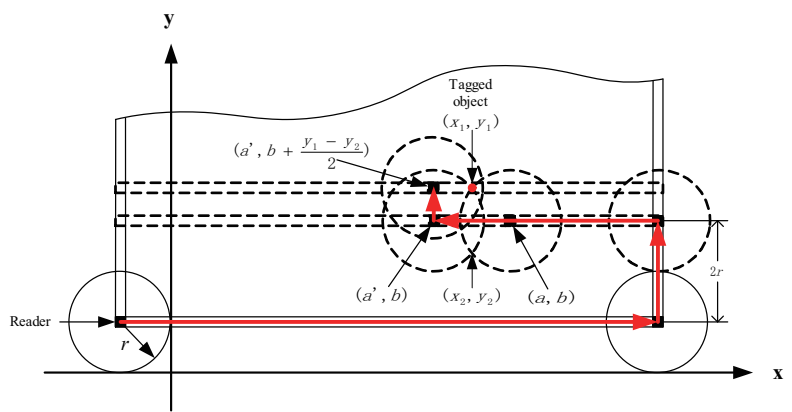

Fig. 2. An example of the continuous scanning.

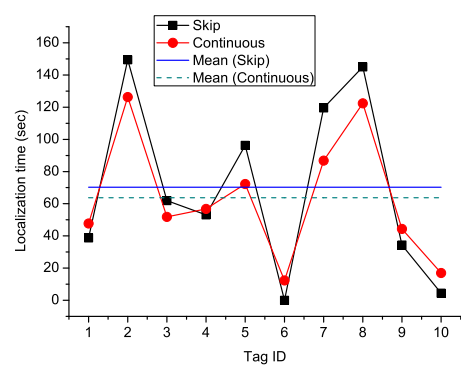

Fig. 3. Localization time.

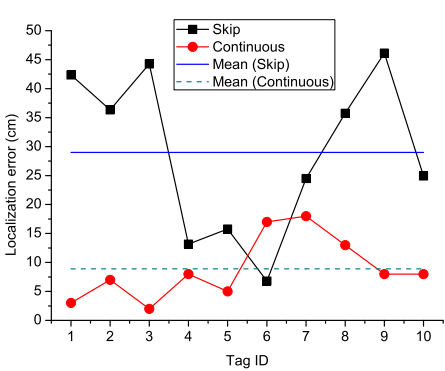

Fig. 4. Localization accuracy.

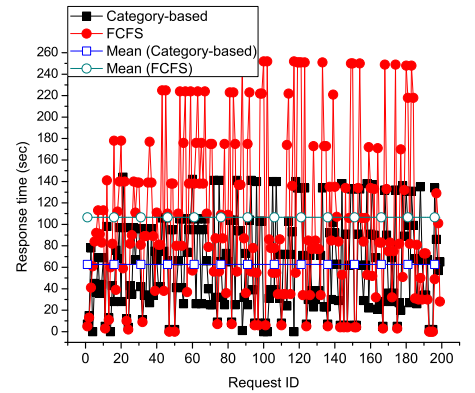

Fig. 5. Time cost. by DTSP. We view each storage area to be scanned as a vertex in a complete graph (the reader can move to any storage area), and then find the minimum-weight Hamiltonian cycle in the graph. Suppose that vertex $i, v_{i}$, has predetermined entry and exit where the reader enters and leaves $v_{i}$. We define the weight between $v_{i}$ and $v_{j}, d_{i j}$, as the time cost that the reader takes to move from $v_{i}$ 's exit to $v_{j}$ 's entry. For example, if $v_{i}$ 's exit and $v_{j}$ 's entry are $\left(x_{1}, y_{1}\right)$ and $\left(x_{2}, y_{2}\right)$ respectively, then we have $d_{i j}=\frac{\left|x_{1}-x_{2}\right|}{v_{x}}+\frac{\left|y_{1}-y_{2}\right|}{v_{y}}$. Assume that there are totally $n(t)$ storage areas at time $t$, we can use a weight matrix to describe the "dynamic" characteristics of vertices $D(t)=\left\{d_{i j}\right\}_{n(t) \times n(t)}$. Given a complete and undirected graph $G(t)=(V(t), E(t))$ with $n(t)$ vertices and a weight matrix $D(t)$, we try to find a minimum-weight path (Hamiltonian cycle) in $G(t)$ at time $t$. The objective function is defined as follows:

$$
\min \left(\sum_{i=1}^{n(t)-1} d\left(\sigma_{i}, \sigma_{i+1}\right)+d\left(\sigma_{n}, \sigma_{1}\right)\right)
$$

where $\sigma(t)$ is a permutation over the set $\{1,2, \ldots, n(t)\}$. The category-based scheduling is a practical solution to DTSP. Such an algorithm samples $G(t)$ at regular time intervals and finds the TSP tour for each $G(t)$ by applying the 2approximation algorithm.

\section{PERformance EVAluation}

Figure 3 and 4 show that the continuous scanning is more efficient than the skip scanning. The mean localization time for the continuous scanning is around $10 \%$ lower than that for the skip scanning. The mean localization error for the continuous scanning is about $60 \%$ of that for the skip scanning. Figure 5 depicts that the category-based scheduling can outperform the
FCFS scheduling. The average response time for the categorybased scheduling is only 63 seconds while that for the FCFS scheduling is 107 seconds.

\section{CONCLUSION}

We build an effective architecture, which consists of two guide rails, and propose two novel algorithms, continuous scanning and category-based scheduling, for locating passive RFID tagged objects. Our theistical experimental results show that the proposed architecture and algorithms can reduce system cost and improve localization efficiency.

\section{ACKNOWLEDGMENT}

This work is supported by China Postdoctoral Science Foundation under Grant No. 2012M521684 and 2013T60849; Fundamental Research Funds for Central Universities under Grant No. ZYGX2014J054, ZYGX2013J083 and 2014QNA5012; National Science Foundation of China under Grant No. F020301, F020803 and 61402404; National Social Science Foundation under Grant No. 15XJY008.

\section{REFERENCES}

[1] L. M. Ni, Y. Liu, Y. C. Lau, and A. P. Patil, "Landmarc: indoor location sensing using active rfid," in PerCom 2003: Proceedings of the IEEE International Conference on In Pervasive Computing and Communications, March 2003, pp. 407-415.

[2] D. Zhang, J. Ma, Q. Chen, and L. M. Ni, "An rf-based system for tracking transceiver-free objects," in PerCom 2007: Proceeding of the 5th Annual IEEE International Conference on Pervasive Computing and Communications, March 2007, pp. 135-144.

[3] C. Wang, H. Wu, and N. F. Tzeng, "Rfid-based 3-d positioning schemes," in INFOCOM 2007: Proceedings of the 26th IEEE International Conference on Computer Communications, 2007, pp. 1235-1243. 